\title{
Traumatisme du coude chez l'enfant, la fracture du condyle latéral
}

\section{Elbow Fracture in Children, Lateral Condyle Fracture}

\author{
V. Ruche $\cdot$ J. Gouverneur $\cdot$ R. Mongiat \\ Reçu le 6 juillet 2017; accepté le 19 octobre 2017 \\ (C) SFMU et Lavoisier SAS 2017
}

Une enfant de six ans se présente au service d'accueil des urgences pour prise en charge d'un gros coude gauche douloureux avec une impotence fonctionnelle, suite à un traumatisme survenu dans un trampoline. L'enfant est tombée sur la paume de la main gauche et il n'y a pas eu de traumatisme direct au niveau du coude. Un mécanisme coude en extension est suspecté. À l'examen clinique, il est retrouvé un gros coude avec une douleur prédominant à la face latérale de cette articulation. L'examen vasculonerveux est strictement normal. Le revêtement cutané est intact. Les articulations sus- et sous-jacentes (épaule et poignet) sont indolores. Les clichés radiologiques du coude gauche face et profil (Fig. 1) objectivent une fracture du condyle latéral, peu déplacée (grade 2 de la classification de Lagrange et Rigault) et de type 2 selon la classification de Milch. Après avis chirurgical, l'enfant est hospitalisée afin de bénéficier d'un traitement chirurgical : réduction et ostéosynthèse par broches. Les suites opératoires sont simples et l'enfant regagne son domicile avec une immobilisation type plâtre brachio-anté-brachio-palmaire et une consultation de contrôle à sept jours.

La fracture du condyle latéral du coude chez l'enfant représente $17 \%$ des fractures de l'extrémité distale de l'humérus. Les autres types de fractures, par ordre de fréquence décroissante, sont: les fractures supracondyliennes (très fréquentes), les fractures de l'épicondyle médial (14\%), les fractures du condyle médial ( 1 à $2 \%$ ) et les fractures de l'épicondyle latéral (très rare) [1]. L'âge moyen de survenue est de 6 à 8 ans. Il s'agit d'une fracture intra-articulaire. Le trait de fracture débute au niveau de la corticale latérale de la région métaphysaire, juste au-dessus du capitulum, puis se dirige vers la trochlée. En fonction de la localisation de l'extrémité médiale du trait de fracture, Milch distingue

\footnotetext{
V. Ruche $(\bowtie) \cdot$ J. Gouverneur $\cdot$ R. Mongiat

Service des urgences,

centre hospitalier Geneviève de Gaulle Anthonioz,

rue du Dr Schweitzer, 52100 Saint-Dizier, France

e-mail : valerie.ruche@ch-saintdizier.fr
}

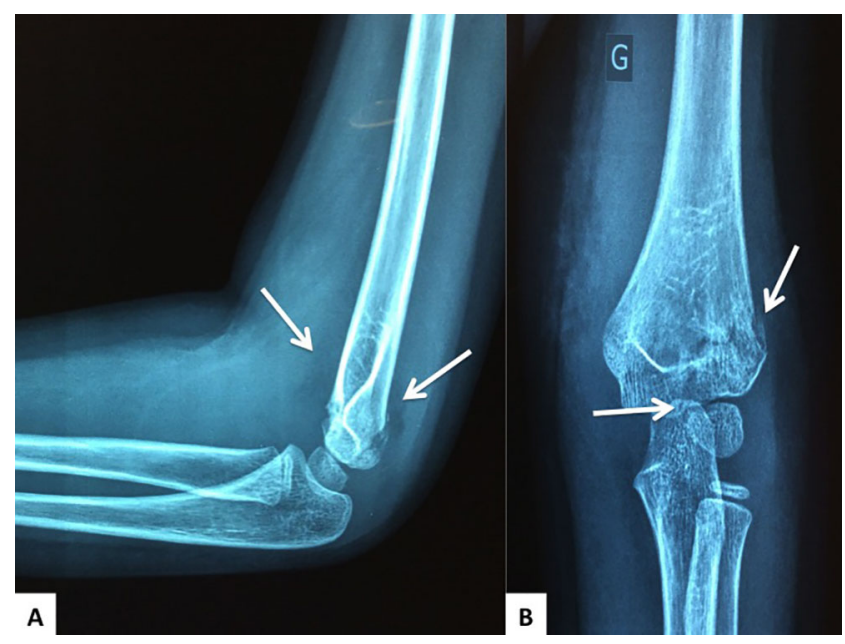

Fig. 1 Radiographies profil (A) et face (B) du coude gauche. A : déplacement des lignes graisseuses péri-articulaires témoignant d'une hémarthrose (flèches blanches) ; B : fracture du condyle latéral avec trait au niveau de la trochlée (flèches blanches)

deux types (type 1 : trait en dehors de la trochlée; type 2 : trait au niveau de la trochlée). La mauvaise réputation de cette fracture tient au fait que, chez le petit enfant, le trait de fracture est non visualisé car les structures ne sont pas encore opacifiées et que le risque de déplacement secondaire de la fracture est important même en cas de fracture non déplacée. La classification la plus souvent utilisée permettant de décrire le déplacement est celle de Lagrange et Rigault qui comporte trois grades (grade 1 : déplacement inférieur à $2 \mathrm{~mm}$, la surface articulaire est peu ou pas modifiée ; grade 2 : déplacement supérieur à $2 \mathrm{~mm}$, la surface articulaire est atteinte ; grade 3 : grand déplacement avec rotation) [2]. Le traitement est souvent chirurgical avec ostéosynthèse par broches. En cas de fracture non déplacée, un traitement orthopédique peut s'envisager mais implique une recherche systématique de déplacement secondaire à sept jours avec réalisation d'une imagerie en coupe type IRM de préférence [3]. 
Liens d'intérêts : Les auteurs déclarent n'avoir aucun lien d'intérêt.

\section{Références}

1. Leroux J, Bernadini I, Abu Amara S, Lechevallier J (2016) Fractures de l'extrémité distale de l'humérus chez l'enfant. EMC (Elsevier Masson SAS, Paris) Appareil locomoteur 11:1-11
2. Lagrange J, Rigault P (1962) Les fractures de l'extrémité inférieure de l'humérus de l'enfant. Rev Chir Orthop 48:334-414

3. Thévenin-Lemoine C, Salanne S, Pham T, et al (2017) Relevance of MRI for management of non-displaced lateral humeral condyle fractures in children. Orthop Traumatol Surg Res 103:777-81 